\title{
A Genetic Algorithm for Solving a Special Class of Nonlinear Bilevel Programming Problems ${ }^{\star}$
}

\author{
Hecheng $\mathrm{Li}^{1,2}$ and Yuping Wang ${ }^{1}$ \\ ${ }^{1}$ School of Computer Science and Technology, \\ Xidian University, Xi'an, 710071, China \\ ${ }^{2}$ School of Science, Xidian University, Xi'an, 710071, China \\ lihecheng@qhnu.edu.cn, ywang@xidian.edu.cn
}

\begin{abstract}
A special nonlinear bilevel programming problem (BLPP), whose follower-level problem is a convex programming with a linear objective function in $y$, is transformed into an equivalent single-level programming by using Karush-Kuhn-Tucker (K-K-T) conditions. To solve the equivalent problem effectively, a new genetic algorithm is proposed. First, a linear programming (LP) is constructed to decrease the dimensions of the transformed problem. Then based on a constraint-handling scheme, a second-phase evolving process is designed for some offspring of crossover and mutation, in which the linear property of follower's function is used to generate high quality potential offspring.
\end{abstract}

Keywords: Bilevel programming problems, genetic algorithm, linear programming problem, constraint handling, optimal solutions.

\section{Introduction}

The bilevel programming problem (BLPP) is a mathematical model of the leaderfollower game. As an optimization problem with a hierarchical structure, BLPP has a wide variety of applications 1. However, owing to the complex structure, the vast majority of research on BLPP is concentrated on the linear version of the problem, and a few works on the nonlinear BLPP 2. Moreover, most of existing algorithms for nonlinear BLPP are usually based on the assumption that all of functions are convex and twice differentiable 3. In recent years, genetic algorithms (GAs) have been used for solving BLPP 2146]. 2] proposed an evolutionary algorithm for solving the BLPP in which the follower's problems are convex. In this paper, we further discuss the simplified model of the BLPP given in [2, in which the follower's objective function is linear in $y$. We construct an LP to avoid increasing the dimensions of the search space, and design a second-phase evolving process after both crossover and mutation.

Since a convex programming can be transformed into another convex programming with a linear objective function, as a result, the proposed algorithm can also be used for solving the BLPP given in 2 .

\footnotetext{
* This work is supported by the National Natural Science Foundation of China (No. 60374063).
} 


\section{Transformation of the Problem}

We consider the following nonlinear bilevel programming problem (BLPP):

$$
\left\{\begin{array}{l}
\min _{x \in X} F(x, y) \\
\text { s.t. } G(x, y) \leq 0 \\
\min _{y \in Y} f(x, y)=c(x)^{T} y \\
\text { s.t. } g(x, y) \leq 0
\end{array}\right.
$$

where $F: R^{n} \times R^{m} \rightarrow R, G: R^{n} \times R^{m} \rightarrow R^{p}, g: R^{n} \times R^{m} \rightarrow R^{q}$, and $c: R^{n} \rightarrow R^{m}$. For $x$ fixed, each component of $g$ is convex and differentiable in $y$. Let the search space $\Omega=\{(x, y) \mid x \in X, y \in Y\}$, and the constraint region $S=\{(x, y) \in \Omega \mid G(x, y) \leq 0, g(x, y) \leq 0\}$. For other related definitions, refer to 23 .

We assume that int $\$ \neq \phi$. Replace the follower's programming problem by K-K-T conditions, we can transform the nonlinear BLPP (1) as follows:

$$
\left\{\begin{array}{c}
\min _{x, y, \lambda} F(x, y) \\
\text { s.t. } G(x, y) \leq 0, g(x, y) \leq 0, \lambda \geq 0 \\
c(x)+\left(\nabla_{y} g(x, y)\right)^{T} \lambda=0, \lambda^{T} g(x, y)=0
\end{array}\right.
$$

where $\lambda=\left(\lambda_{1}, \lambda_{2}, \ldots, \lambda_{q}\right)^{T}$ is Lagrangian multipliers.

\section{Constraint-Handling and Decreasing the Dimensions of the Transformed Problem}

For any infeasible individual $B$, a new constraint-handling scheme is designed to generate an approximate feasible individual $D$. Firstly, randomly choose an individual $A \in S$. Let $\hat{S}=\left\{(x, y) \in \Omega \mid G(x, y) \leq \epsilon_{1}, g(x, y) \leq \epsilon_{2}\right\}$, and $\epsilon=$ $\left(\epsilon_{1}^{T}, \epsilon_{2}^{T}\right)^{T}$, where $\epsilon_{i}$ are small positive vectors and tend to zero with the increasing of the generations. Let $D=r B+(1-r) A$, where $r \in(0,1)$ is random number, if $D \in \hat{S}$, then stop. Otherwise, let $B=D$, and re-compute $D$. The process is repeated until $D \in \hat{S}$.

For fixed $\bar{x}$ and $\bar{y}$, one can get $\lambda$ by solving the following LP:

$$
\left\{\begin{array}{c}
\bar{u}(\bar{x}, \bar{y})=\min _{\lambda, U}(1,1, \ldots, 1) U \\
\text { s.t. } h(\bar{x}, \bar{y}, \lambda)+U=0 \\
\lambda \geq 0, U \geq 0
\end{array}\right.
$$

where $h(\bar{x}, \bar{y}, \lambda)=\left(\left(c(\bar{x})+\left(\nabla_{y} g(\bar{x}, \bar{y})\right)^{T} \lambda\right)^{T}, \lambda^{T} g(\bar{x}, \bar{y})\right)^{T}, U$ is an artificial vector. Thus we only need to evolve $(\bar{x}, \bar{y})$ in the algorithm, this is equivalent to the reduction of the problem dimensions. 


\section{Proposed Algorithm(Algorithm 1)}

Step 1 (Initialization). Randomly generate the initial population $\operatorname{pop}(0)$ of $N_{p}$ points in $\Omega$ such that there is at least one point in $S$. Apply the constrainthandling scheme to the points which don't belong to $\hat{S}$ such that these $N_{p}$ points are in $\hat{S}$. Denote $N=\{(x, y) \in \operatorname{pop}(0) \cap S\}$ and let $k=0$.

Step 2. Evaluate the fitness $\bar{F}(x, y)=\left\{\begin{array}{ll}F(x, y), & \bar{u}(x, y)=0 ; \\ K+\mu \bar{u}(x, y), & \bar{u}(x, y) \neq 0 .\end{array}\right.$ where $K$ is an upper-bound of $F(x, y)$ on the set $\{(x, y) \mid \bar{u}(x, y)=0\}, \mu \geq 0$.

Step 3 (Crossover). For each pair of randomly matched parents $p_{1}$ and $p_{2}$, the crossover generates offspring: $o 1=r p_{1}+(1-r) p_{2}, o 2=(1-r) p_{1}+r p_{2}$, where $r \in[0,1]$ is random number. Let $O 1$ stands for the set of all these offspring.

Sept 4 (Mutation). Gaussian mutation is executed, and the offspring set is denoted by $O 2$.

Step 5 (Constraint-handling). Let $O=O 1 \cup O 2$. If any point in $O$ is not in $S$, then arbitrarily choose $\eta \in N$ to replace a point in $O$. Apply the proposed constraint-handling method to modify each point $\tau \in O$ which is not in $\hat{S}$, such that all points in $O$ are in $\hat{S}$. Let $N=\{(x, y) \in S \cap O\}$ and $\epsilon=\theta \epsilon, \theta \in[0,1]$.

Step 6 (Improving offspring by the second-phase evolving). For each point $(x, y) \in$ $N \subset O$, let $d$ be a descent direction of $f(x, y)$ in $y$ for $x$ fixed. Take $\rho>0$ such that $\bar{y}=y+\rho d$ reaches the boundary of the feasible region of the follower's problem for $x$ fixed. Replace $(x, y)$ by $(x, \bar{y})$ in $O$.

Step 7 (Selection). Evaluate the fitness values of all points in $O$. Select the best $n_{1}$ points from the set $\operatorname{pop}(k) \cup O$ and randomly select $N_{p}-n_{1}$ points from the remaining points of the set. All these selected points form the next population $\operatorname{pop}(k+1)$.

Step 8. If the termination condition is satisfied, then stop; Otherwise, let $k=$ $k+1$, go to Step 3 .

\section{Simulation Results}

In this section, 10 benchmark problems $F 1-F 10$ are selected from the references 34 4567 for simulation. In order to demonstrate the effectiveness of the proposed algorithm on the BLPPs with nondifferentiable leader level functions, we construct two benchmark problems $F 11$ and $F 12$ only by replacing the leader's objective functions in $F 8$ and $F 10$ by $F(x, y)=\left|\sin \left(2 x_{1}+2 x_{2}-3 y_{1}-3 y_{2}-60\right)\right|$ and $\min _{x} F(x, y)=\left|\sin \left(\left(x_{1}-30\right)^{2}+\left(x_{2}-20\right)^{2}-20 y_{1}+20 y_{2}-225\right)\right|$, respectively.

The parameters are chosen as follows: $N_{p}=30$, the crossover probability $p_{c}=0.8$, the mutation probability $p_{m}=0.3, n_{1}=10, \mu=1$, the initial $\epsilon=$ $(1, \cdots, 1) \in R^{p+q}, \theta=0.7$ for $k \leq k_{\max } / 2$, while $\theta=0$ for $k>k_{\max } / 2$, where $k$ represents generation number, while $k_{\max }$ the maximum generation number. For $F 1-F 3, F 5, F 8, F 9$ and $F 11, k_{\max }=50$, while for other problems, $k_{\max }=100$ . We execute Algorithm 1 in 30 independent runs on each problem, and record the following data: (1) leader's(follower's) objective values $F\left(x^{*}, y^{*}\right)\left(f\left(x^{*}, y^{*}\right)\right)$ at the best solution; (2) the leader's objective function value $F(\bar{x}, \bar{y})$ at the worst point $(\bar{x}, \bar{y}) ;(3)$ mean value of $F(x, y)$ in all 30 runs(denoted by $F_{\text {mean }}$ in short). 
Table 1. Comparison of the results found by Algorithm 1 and the related algorithms

\begin{tabular}{|c|c|c|c|c|c|c|}
\hline \multirow{2}{*}{ No. } & \multicolumn{2}{|c|}{$F\left(x^{*}, y^{*}\right)$} & \multicolumn{2}{|c|}{$f\left(x^{*}, y^{*}\right)$} & \multirow{2}{*}{} \\
\cline { 2 - 5 } & Algorithm 1 & Ref. & Algorithm 1 & Ref. & $F_{\text {mean }}$ & $F(\bar{x}, \bar{y})$ \\
\hline$F 1[\underline{]}]$ & -9 & $N A$ & -54 & $N A$ & -9 & -9 \\
\hline$F 2[\underline{3}]$ & 2 & 2 & 12 & 12 & 2 & 2 \\
\hline$F 3[\underline{4}]$ & 1000 & 1000 & 1 & 1 & 1000 & 1000 \\
\hline$F 4[\underline{4}]$ & -1.2098 & 3.57 & 7.617 & 2.4 & -1.2096 & -1.2090 \\
\hline$F 5[\underline{4}]$ & 100.003 & 100.58 & 0 & 0.001 & 100.012 & 100.039 \\
\hline$F 6[\underline{4}]$ & 81.3262 & 82.44 & -0.3198 & 0.271 & 81.3263 & 81.3266 \\
\hline$F 7[\underline{6}]$ & 0 & 0 & 5 & 5 & 0 & 0 \\
\hline$F 8[\underline{7}]$ & 0 & 5 & 200 & 0 & 0 & 0 \\
\hline$F 9[\underline{6}]$ & 469.1429 & 469.1429 & 8.8571 & 8.8571 & 469.1429 & 469.1429 \\
\hline$F 10[\underline{8}]$ & 225 & 225 & 100 & 100 & 225 & 225 \\
\hline$F 11$ & 0 & $N A$ & 200 & $N A$ & 0 & 0 \\
\hline$F 12$ & 0 & $N A$ & 100 & $N A$ & 0 & 0 \\
\hline
\end{tabular}

All results are presented in Table 1, where NA means that the result is not available for the algorithms and Ref. stands for the related algorithms in references. It can be seen from Table 1 that for $F 4, F 5, F 6$ and $F 8$, the best results found by Algorithm 1 are better than those by the compared algorithms. For F11 and F12, Algorithm1 found the optimal solutions. For other problems, the best results found by Algorithm1 are almost as good as those by the compared algorithms.

\section{References}

1. Colson, B., Marcotte, P., Savard, G: Bilevel programming: A survey. A Quarterly Journal of Operations Research(4OR) 3(2005) 87-107

2. Wang, Yuping, Jiao, Yong-Chang, Li, Hong : An Evolutionary Algorithm for Solving Nonlinear Bilevel Programming Based on a New Constraint-Handling Scheme. IEEE Transactions on Systems, Man, and Cybernetics(C) 35(2)(2005) 221-232

3. Bard, J. F.: Practical Bilevel Optimization. Norwell, Kluwer, MA (1998)

4. Oduguwa, V., Roy, R.: Bi-level optimization using genetic algorithm. Proceeds of the 2002 IEEE International Conference on Artificial Intelligent Systems (ICAIS02) (2002) 123-128

5. Zheng, P.-E.: Hierarchical optimization algorithm-based solutions to a class of bilevel programming problems. Systems Engineering and Electronics 27(4) (2005) 663-665

6. Li, H., Wang, Yuping: A hybrid genetic algorithm for nonlinear bilevel programmings. J. Xidian University 29(6) (2002) 840-843

7. Aiyoshi, E., Shimuzu, K.: A solution method for the static con-strained Stackelberg problem via penalty method. IEEE Trans. Autom. Control AC-29(12) (1984) 1112-1114

8. Shimizu, K., Aiyoshi, E.: A new computational method for Syackelberg and minmax problems by use of a penalty method. IEEE Trans. Autom. Control AC-26(2) (1981) 460-466 